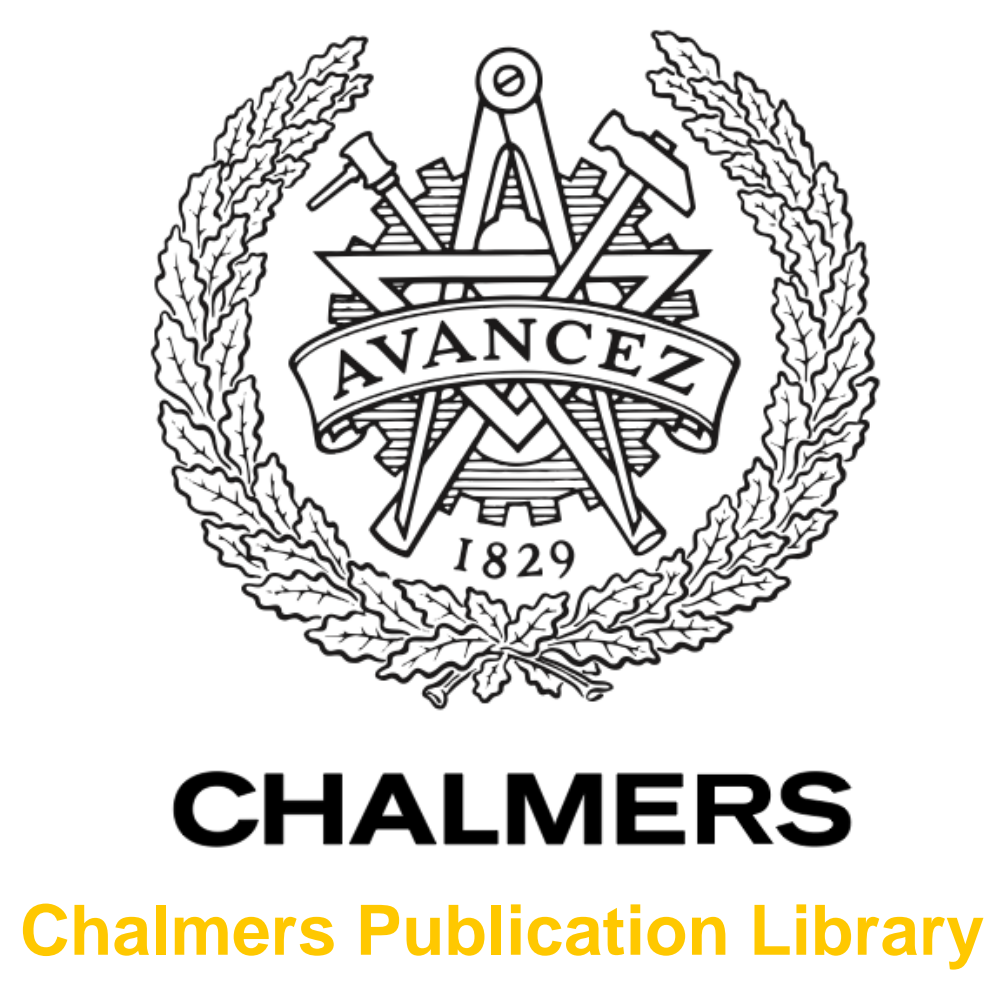

\title{
A Tighter Upper Bound on the Capacity of the Nondispersive Optical Fiber Channel
}

This document has been downloaded from Chalmers Publication Library (CPL). It is the author's version of a work that was accepted for publication in:

European Conference on Optical Communication (ECOC), 2017.

Citation for the published paper:

Keykhosravi, K. ; Durisi, G. ; Agrell, E. (2017) "A Tighter Upper Bound on the Capacity of the Nondispersive Optical Fiber Channel". European Conference on Optical Communication (ECOC), 2017.

Downloaded from: http://publications.lib.chalmers.se/publication/250961

Notice: Changes introduced as a result of publishing processes such as copy-editing and formatting may not be reflected in this document. For a definitive version of this work, please refer to the published source. Please note that access to the published version might require a subscription. 


\title{
A Tighter Upper Bound on the Capacity of the Nondispersive Optical Fiber Channel
}

\author{
Kamran Keykhosravi, Giuseppe Durisi, and Erik Agrell \\ Department of Electrical Engineering, Chalmers University of Technology, kamrank@chalmers.se
}

\begin{abstract}
An upper bound on the capacity of the nondispersive optical fiber channel is presented. This bound, which is valid for arbitrary launch powers, confines the capacity within a much narrower range compared to what the previously known upper bound provided.
\end{abstract}

\section{Introduction}

Due to the ever-increasing data demand caused by emerging bandwidth-hungry services, such as internet-of-thing applications and cloud computing, current optical systems are reaching their limits and a capacity crunch is imminent. To utilize the existing optical networks more effectively, it is necessary to study the capacity of the fiberoptical channel. Many lower bounds on capacity are available ${ }^{1}$; most of them rely on the theory of mismatched decoding. All the available lower bounds on the capacity of the dispersive and nonlinear fiber-optical channel saturate or fall to zero in the high-power regime. However, the only known upper bound ${ }^{2}$ increases to infinity with power.

The capacity analysis of the fiber-optical channel governed by the nonlinear Schrödinger (NLS) equation is, in general, cumbersome because the input-output relation is given implicitly through a partial differential equation. For the case of nondispersive fiber-optical systems, however, the discrete-time channel between the samples of the transmitted and the received signals can be described by a conditional probability distribution function (pdf) that is known in a closed form ${ }^{3-5}$. By studying this conditional pdf, multiple lower bounds on the per-sample capacity have been obtained $^{3,4}$. However, the only known nonasymptotic upper bound on the capacity of the nondispersive NLS channel is too loose to provide any insight about the tightness of these lower bounds at medium and high powers.

In this paper, we present a nonasymptotic upper bound on the per-sample capacity of the nondispersive NLS channel using techniques similar to those employed in ${ }^{6,7}$. We evaluate the upper bound for a 5000-km nondispersive NLS channel with distributed amplification, whose parameters can be found in Table 1, and compare it with the known lower bounds. The results are shown in Figure 1: a significant improvement can be observed compared to the previously known upper bound.

\section{Channel Model}

The nondispersive NLS channel with ideal distributed amplification can be modeled by the NLS equation by setting the dispersion to zero, which results in the partial differential equation

$$
\frac{\partial \mathbf{a}(z, t)}{\partial z}-j \gamma|\mathbf{a}(z, t)|^{2} \mathbf{a}(z, t)=\mathbf{n}(z, t) .
$$

Here, $\mathbf{a}(z, t)$ is the baseband optical signal at time $t$ and location $z$, the nonlinear coefficient is denoted by $\gamma$, and $\mathbf{n}(z, t)$ is a complex Gaussian noise process with autocorrelation

$$
\mathrm{E}\left[\mathbf{n}(z, t) \mathbf{n}^{*}\left(z^{\prime}, t^{\prime}\right)\right]=N_{0} \delta_{W_{N}}\left(t-t^{\prime}\right) \delta\left(z-z^{\prime}\right) .
$$

In (2), we used $\delta(\cdot)$ to denote the Dirac delta function and $\delta_{W_{N}}(x)=W_{N} \operatorname{sinc}\left(W_{N} x\right)$, where $W_{N}$ is the noise bandwidth. The noise power spectral density is $N_{0}=n_{\mathrm{sp}} h \nu \alpha$. A list of all parameters is provided in Table 1. We denote the total noise variance of the samples at the receiver by $P_{N}=2 W_{N} N_{0} L$ where $L$ is the fiber length. The discrete-time channel between the samples of the transmitted signal $\mathbf{a}(0, t)$ and the received signal $\mathbf{a}(L, t)$ can be described by the following conditional pdf in the polar coordinate system: ${ }^{5}$

$$
\begin{aligned}
f_{\mathbf{r}, \boldsymbol{\theta} \mid \mathbf{r}_{0}, \boldsymbol{\theta}_{0}}\left(r, \theta \mid r_{0}, \theta_{0}\right)=\frac{f_{\mathbf{r} \mid \mathbf{r}_{0}}\left(r \mid r_{0}\right)}{2 \pi} \\
+\frac{1}{\pi} \sum_{m=1}^{\infty} C_{m}\left(r, r_{0}\right) e^{j m\left(\theta-\theta_{0}\right)} .
\end{aligned}
$$

Here, $r$ and $\boldsymbol{\theta}$ are the amplitude and the phase of the received signal and $\mathbf{r}_{0}$ and $\boldsymbol{\theta}_{0}$ are those of the transmitted one. The conditional pdf $f_{\mathbf{r} \mid \mathbf{r}_{0}}\left(r \mid r_{0}\right)$ of $\mathbf{r}$ given $\mathbf{r}_{0}$ as well as the Fourier coefficients 
Tab. 1: System parameters and their description

\begin{tabular}{ccc}
\hline \hline Symbol & Value & Meaning \\
\hline$n_{\mathrm{sp}}$ & 1 & Emission factor \\
$h \nu$ & $1.28 \cdot 10^{-19} \mathrm{~J}$ & Photon energy \\
$\alpha$ & $0.2 \mathrm{~dB} / \mathrm{km}$ & Fiber attenuation \\
$\gamma$ & $1.27(\mathrm{~W} \mathrm{~km})^{-1}$ & Nonlinear coefficient \\
$W_{N}$ & $32 \mathrm{GHz}$ & Noise bandwidth \\
\hline$P_{N}$ & $-27.2 \mathrm{dBm}$ & Total noise variance \\
\hline \hline
\end{tabular}

$C_{m}\left(r, r_{0}\right)$ can be found in ${ }^{5}$.

The discrete-time NLS channel can also be described by the split-step Fourier method, ${ }^{8}$ according to which (1) is approximated by a cascade of $K$ subchannels. Specifically, let $\mathbf{x}_{0}$ denote the channel input. The channel output $x_{K}$ is obtained by iterating

$$
\mathbf{x}_{k+1}=\mathbf{x}_{k} e^{j L \gamma\left|\mathbf{x}_{k}\right|^{2} / K}+\mathbf{n}_{k+1}
$$

where $\mathbf{n}_{k+1} \sim \mathcal{C N}\left(0, P_{N} / K\right)$ for $k=$ $0,1, \ldots, K-1$. The output $\mathbf{x}_{K}$ of the splitstep Fourier method approximates accurately the samples of $\mathbf{a}(L, t)$ when $K \rightarrow \infty$, i.e., when the step size vanishes.

Since the channel (3) is memoryless, its capacity, $\mathcal{C}$ (in bits per channel use), can be evaluated by calculating the maximum of the mutual information between the channel input and output, $I\left(\mathbf{r}, \boldsymbol{\theta} ; \mathbf{r}_{0}, \boldsymbol{\theta}_{0}\right)$, over all the distributions that satisfy the power constraint $\mathrm{E}\left[\mathbf{r}_{0}^{2}\right] \leq P$. Next, we list some known bounds on $\mathcal{C}$ and then propose a novel upper bound.

\section{Bounds on the Channel Capacity}

Since the conditional pdf describing the channel law is known, lower bounds on the per-sample capacity of the nondispersive NLS channel can be computed by fixing the input distribution and evaluating the resulting mutual information via numerical integration. Yousefi et al. ${ }^{3}$ obtained a lower bound by considering a multiple-ring input constellation and by optimizing radius and probability of each ring under a power constraint. However, optimizing this distribution is computationally expensive and becomes impractical at high powers. Next, three lower bounds are presented. Afterwards we shall review the only known upper bound, and then introduce our novel upper bound.

Lower Bound 1: This bound relies on a circularly-symmetric input distribution with halfGaussian amplitude profile ${ }^{3}$, i.e,

$$
f_{\mathbf{r}_{0}}\left(r_{0}\right)=\sqrt{2 /(\pi P)} \exp \left(-r_{0}^{2} /(2 P)\right) .
$$

It was shown that this lower bound is tight when the SNR goes to infinity ${ }^{3}$.

Lower Bound 2: This lower bound, which has not been evaluated for this channel previously, is obtained by considering a circularly-symmetric Gaussian input distribution, which is capacityachieving in the absence of nonlinearity. This lower bound is tight in the low-power regime where the nonlinearity is weak.

Lower Bound 3: Turitsyn et al. ${ }^{4}$ developed a lower bound on the per-sample capacity that can be calculated for any input power by means of numerical integration. This bound can be achieved by a noncoherent receiver; indeed, only the statistics of the output's amplitude was used in its derivation.

Previous Upper Bound: The capacity of a dispersive discrete-time NLS channel is no larger than $\log \left(1+\right.$ SNR) (bits per channel use). ${ }^{2}$ This bounds holds also in the nondispersive case.

New Upper Bound: We show that the capacity of the nondispersive NLS channel (1) is upperbounded as

$$
\begin{aligned}
\mathcal{C} \leq & \min _{\lambda>0, \alpha>0}\left\{\alpha \log \left(\frac{P+P_{N}}{\alpha}\right)+\log (\pi \Gamma(\alpha))\right. \\
& \left.+\lambda+\max _{r_{0}>0}\left\{g_{\lambda, \alpha}\left(r_{0}, P\right)\right\}\right\}
\end{aligned}
$$

where

$$
\begin{aligned}
& g_{\lambda, \alpha}\left(r_{0}, P\right)=(\alpha \log e-\lambda) \frac{r_{0}^{2}+P_{N}}{P+P_{N}} \\
& -h\left(\mathbf{r} \mid \mathbf{r}_{0}=r_{0}\right)+(1-2 \alpha) \mathrm{E}\left[\log (\mathbf{r}) \mid \mathbf{r}_{0}=r_{0}\right] \\
& -h\left(\boldsymbol{\theta} \mid \mathbf{r}, \mathbf{r}_{0}=r_{0}, \boldsymbol{\theta}_{0}=0\right) .
\end{aligned}
$$

Here, $h(\cdot \mid \cdot)$ is the conditional differential entropy function. The term $h\left(\mathbf{r} \mid \mathbf{r}_{0}=r_{0}\right)$ can be computed numerically using $f_{\mathbf{r} \mid \mathbf{r}_{0}}\left(r \mid r_{0}\right)$. Furthermore, $h\left(\boldsymbol{\theta} \mid \mathbf{r}, \mathbf{r}_{0}=r_{0}, \boldsymbol{\theta}_{0}=0\right)$ can be computed numerically using (3) and Bayes' theorem to find $f_{\boldsymbol{\theta} \mid \mathbf{r}, \mathbf{r}_{0}, \boldsymbol{\theta}_{0}}\left(\theta \mid r, r_{0}, \theta_{0}\right)$.

The proof of (6) relies on a dual formula for channel capacity ${ }^{9}$; furthermore, a Gamma distribution with parameters $\alpha>0$ and $\beta=\left(P+P_{N}\right) / \alpha$ is chosen as auxiliary pdf of the random variable $\mathbf{r}^{2}$ to upper-bound the output's differential entropy. The method of Lagrange multipliers is used to replace the maximization over the set of input distributions satisfying the power constraint, with a maximization over $r_{0} \geq 0$. Also, the symmetry of the channel with respect to the input phase $\theta_{0}$ (see (3)) is exploited to avoid an optimization over 
the phase of the input signal.

\section{Numerical Example}

We evaluate our novel capacity upper bound and the available upper and lower bounds for a nondispersive $5000-\mathrm{km}$ fiber-optical system with ideal distributed amplification. The channel parameters can be found in Table 1. Figure 1 depicts the previous and new upper bounds together with the three lower bounds on the capacity, described in the previous section. We see that, in the low-power regime, the per-sample capacity of the nondispersive NLS channel is close to that of the AWGN channel (previous upper bound). This is expected, because the nonlinearity is weak at low powers. One can also observe that lower bound 2 is tighter than lower bound 1 at low powers, as expected (see the magnified figure).

Figure 1 suggests that the slope of capacity start decreasing at moderate powers. This can be explained as follows. We see from (4) that, at each step, the signal experiences a nonlinear phase shift proportional to its amplitude square. This phase distortion is a nonlinear function of both the transmitted signal and the noise, and increases with the input power. This means that, as the power increases, the amount of information that can be conveyed through the phase of the transmitted signal decreases, which determines a reduction in the slope of capacity. The effects of the phase noise becomes noticeable at about $0 \mathrm{dBm}$. At high powers (around $30 \mathrm{dBm}$ ), the phase of the received samples converges to a uniform distribution over $[0,2 \pi)$ and becomes essentially independent of the transmitted signal. This means that information can be conveyed only through the input's amplitude. This is confirmed by the fact that capacity seems to grow with the same slope as that of lower bound 3, which relies on noncoherent detection.

\section{Conclusion}

A nonasymptotic upper bound on the capacity of the nondispersive fiber-optical channel was introduced. We evaluated the upper bound together with two known lower bounds ${ }^{3,4}$. We also evaluated the previously known upper bound and a lower bound that is tight at low powers. A comparison between these bounds illustrates that the proposed upper bound confines the capacity to a much narrower range than the previously known one. Future work may involve a comparison between the proposed upper bound and the tighter

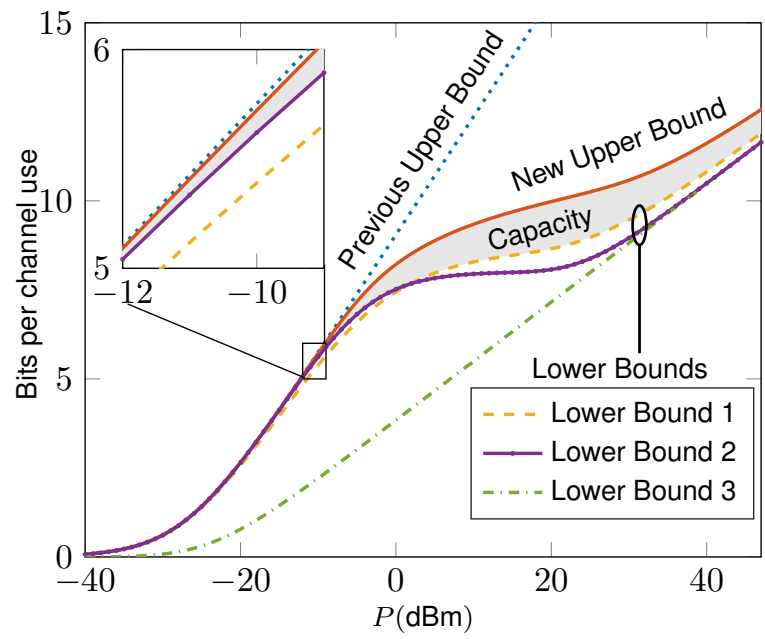

Fig. 1: The proposed upper bound on the capacity of the nondispersive optical fiber channel together with previously known bounds. The capacity is confined to the shaded region.

lower bounds obtainable by optimizing multiplering constellations ${ }^{3,6}$.

\section{Acknowledgment}

The support from the Swedish Research Council (VR) under grant no. 2013-5271 is gratefully acknowledged.

\section{References}

[1] M. Secondini and E. Forestieri, "Scope and limitations of the nonlinear Shannon limit," J. Lightw. Technol., Vol. 35, no. 5, pp. 893-902 (2017).

[2] G. Kramer et al., "Upper bound on the capacity of a cascade of nonlinear and noisy channels," Proc. IEEE Info. Theory Workshop (ITW), Apr.-May (2015).

[3] M. I. Yousefi and F. R. Kschischang, " On the per-sample capacity of nondispersive optical fibers," IEEE Trans. Inform. Theory, Vol. 57, no. 11, pp. 7522-7541 (2011).

[4] K. S. Turitsyn, et al., "Information capacity of optical fiber channels with zero average dispersion," Phys. Rev. Lett., Vol. 91, no. 20, p. 203901 (2003).

[5] K. P. Ho, Phase-Modulated Optical Communication Systems, Springer (2005).

[6] M. Katz and S. Shamai, "On the capacity-achieving distribution of the discrete-time noncoherent and partially coherent AWGN channels," IEEE Trans. Inform. Theory, Vol. 50, no. 10,pp. 2257-2270 (2004).

[7] G. Durisi. "On the capacity of the block-memoryless phase-noise channel," IEEE Commun. Lett., Vol. 16, no. 8, pp. 1157-1160 (2012).

[8] G. P. Agrawal, Nonlinear Fiber Optics, Academic Press (2007).

[9] A. Lapidoth and S. M. Moser, "Capacity bounds via duality with applications to multiple-antenna systems on flatfading channels," IEEE Trans. Inform. Theory, Vol. 49, no. 10, pp. 2426-2467 (2003). 